\title{
3D simulation of the matter transport by surface diffusion within a level-set context
}

\author{
Julien Bruchon* — Daniel Pino Muñoz* — François Valdivieso** \\ Sylvain Drapier* — Guillaume Pacquaut*
}

\author{
* LTDS UMR CNRS 5513 \\ ** PECM UMR CNRS 5146 \\ Ecole Nationale Supérieure des Mines de Saint-Etienne \\ Centre Sciences des Matériaux et des Structures \\ 158 cours Fauriel \\ F-42023 Saint-Etienne cedex 2 \\ bruchon@emse.fr
}

\begin{abstract}
Within the framework of the sintering process simulation, this paper proposes a numerical strategy for the direct simulation of the matter transport by surface diffusion. A level-set method is used to describe the topological changes which arise at the free boundary of the sintering particles. The surface velocity is found to be proportional to the surface Laplacian of the curvature, that is, proportional to the fourth-order derivative of the level-set function. Consequently, both curvature and velocity must be computed carefully and with accuracy. Finally, three-dimensional simulations are shown and investigated.

RÉSUMÉ. Dans le contexte général de la simulation du procédé de frittage, cet article présente une stratégie numérique pour la simulation directe du transport de matière par diffusion surfacique. Une méthode level-set est utilisée pour décrire l'évolution de la surface libre des grains. La vitesse d'interface est alors fonction du Laplacien surfacique de la courbure, i.e. de la dérivée quatrième de la fonction level-set. La courbure et la vitesse surfacique doivent donc être calculées rigoureusement et avec précision. Plusieurs exemples de simulations, en trois dimensions, sont présentés et analysés.

KEYWORDS: surface diffusion, level-set method, curvature, Laplace-Beltrami operator, sintering process

MOTS-CLÉS : diffusion surfacique, méthode level-set, courbure, opérateur de Laplace-Beltrami, procédé de frittage.
\end{abstract}

DOI:10.3166/EJCM.19.281-292 (C) 2010 Lavoisier, Paris

EJCM - 19/2010. Giens 2009, pages 281 to 292 


\section{Introduction}

The general context of this work is the direct simulation of the formation as well as of the subsequent evolution of a powder compact microstructure during a sintering process. Such a process consists in consolidating a metallic or ceramic powder compact, by thermally activating diffusion phenomena to a temperature lower than the melting temperature of the material. When considering a pressure-free sintering process (see (Ashby, 1974) for further details), the surface diffusion is the path of matter transport which is first activated (see Figure 1). Indeed, in order to minimize the surface energy, the matter flows over the grain free boundary towards the maximum (in absolute value) of curvature. The location of the grain centres remains unchanged under this transport path, that is, the porosity does not decrease (no shrinkage). Due to this first diffusion, stresses appear in the material, giving rise to the boundary diffusion and to the volume diffusion, with a subsequent decrease of the global porosity.

This paper deals with the matter transport by the surface diffusion from the grain surface towards the neck formed between two grains. This situation does not involve the mechanical response of the material, since the diffusion is described only by a geometrical criterion: the surface Laplacian of the curvature. An outline of the paper is as follows. The physical description of the surface diffusion is first detailed in Section 2. Section 3 is devoted to the level-set strategy developed to solve the surface diffusion equations. Finally, numerical simulations are shown in Section 4.

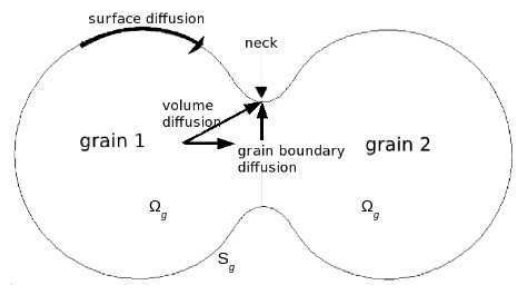

Figure 1. Matter transport between two identical grains

\section{Surface diffusion equations}

Let $\Omega_{g}$ be a set of grains, and let $S_{g}$ be the free boundary of this set (see Figure 1). Regarding the literature about the ceramic sintering process modelling (Ashby, 1974; Bouvard et al., 1996), the matter flow by surface diffusion is characterized by a flux $\mathbf{j}_{\mathbf{s}}$ along the free surface $S_{g}$, driven by chemical potential gradients. In turn, these gradients depend on the gradient of the mean curvature $K$ :

$$
\mathbf{j}_{\mathbf{s}}=-\frac{\delta_{s} D_{s} \gamma_{s}}{R T} \nabla_{\mathrm{s}} K
$$

where $T$ is the absolute temperature, $R$ is the ideal gas constant, $D_{s}$ is the surface diffusion coefficient, $\delta_{s}$ is the thickness in which the diffusion occurs and $\gamma_{s}$ is the 
surface free energy. In expression [1], operator $\nabla_{\mathrm{s}}$ denotes the surface gradient, defined as the tangential component of the gradient

$$
\nabla_{\mathrm{s}} K=\nabla K-(\nabla K \cdot \mathbf{n}) \mathbf{n}
$$

where $\mathbf{n}$ is the outward-pointing unit vector normal to the free surface $S_{g}$. The surface gradient can easily be rewritten by introducing $P$, the projection matrix onto the plane tangent to $S_{g}$ :

$$
\nabla_{\mathrm{s}} K=P \nabla K
$$

with

$$
P=I-\mathbf{n} \otimes \mathbf{n}
$$

where $I$ is the identity matrix.

The surface flux $\mathbf{j}_{\mathbf{s}}$ results in the deposition or removal of material, which gives rise to a displacement rate, assumed to be normal to the surface. This surface diffusion velocity is then written $\mathbf{v}_{\mathbf{s}}=v_{n} \mathbf{n}$. The mass balance between, on one hand the matter which flows over the free surface $S_{g}$, and on the other hand the normal displacement of this surface, leads to the following expression of the surface velocity (Bouvard et al., 1996):

$$
\mathbf{v}_{\mathbf{s}}=-\Omega\left(\nabla_{\mathrm{s}} \cdot \mathbf{j}_{\mathbf{s}}\right) \mathbf{n}=C_{0}\left(\Delta_{\mathrm{s}} K\right) \mathbf{n}
$$

where $\Omega$ is the molar volume of the material and with $C_{0}=\frac{\delta_{s} D_{s} \Omega \gamma_{s}}{R T}$ which is assumed to be constant in this approach. It means that the temperature is assumed to be at least uniform in the computational volume. In fact, all the simulations presented in the following correspond to isothermal cases. The operator $\Delta_{\mathrm{s}}$, the "surface Laplacian" operator is the so-called Laplace-Beltrami operator.

Finally, let us remark that the surface velocity given by Equation [3] preserves the volume. Indeed, if $\left|\Omega_{g}\right|$ denotes the grain volume measure, its variation during the process is expressed by

$$
\frac{d\left|\Omega_{g}\right|}{d t}=\int_{S_{g}} \mathbf{v}_{\mathbf{s}} \cdot \mathbf{n} d S=-\Omega \int_{S_{g}} \nabla_{\mathbf{s}} \cdot \mathbf{j}_{\mathbf{s}} d S
$$

Since the flux is continuous and $S_{g}$ is a closed surface, this variation vanishes, and $d\left|\Omega_{g}\right| / d t=0$. Consequently, the grain volume remains constant. This point, as well as the absence of shrinkage, will be a criterion to assert the relevancy of our simulations.

\section{Level-set formulation}

Equation [3] is discretized by using a finite element method. The simulation of the matter transport by surface diffusion requires only a surface description of the 
grains. However, the final objective of this work is to take into account the different paths of the matter transport, including in volume. That is why the numerical strategy proposed in this paper is based on a volume description of the grains, i.e., the whole computational domain is meshed. Furthermore, in order to deal implicitly with the topological changes arising in the grain surface (formation of the neck), an Eulerian approach is chosen to describe the grain surface changes. Hence, the computational domain $\Omega \subset \mathbb{R}^{3}$ (the unit cube) is made up of two parts: the grain set $S_{g}$, and the surrounding media $\Omega_{a}$ (the air, for example), $\Omega=\Omega_{g} \cup \Omega_{a}$. An additional function, the level-set function, $\phi: \Omega \rightarrow \mathbb{R}$, is then required to describe the grain free boundary. This point is detailed in the next section.

\subsection{Description of the grains}

For any time $t$, and any point $x \in \Omega$, the value of the level-set function $\phi(x, t)$ is positive if $x \in \Omega_{a}(t)$, and is negative if $x \in \Omega_{g}(t)$. Consequently the grain free boundary is defined by the zero isosurface of $\phi$ :

$$
S_{g}(t)=\{x \in \Omega ; \phi(x, t)=0\}
$$

This level-set function $\phi$, which has to be smooth enough in the vicinity of $S_{g}$, is defined as following, according to (Coupez, 2006; Bernacki et al., 2008). At the initial time $t=0, \phi$ is initialized by the grain initial state $\phi_{0}, \phi(x, 0)=\phi_{0}(x)$, with

$$
\phi_{0}(x)=\left\{\begin{array}{lll}
\frac{2 E}{\pi} \sin \left(\frac{\pi d\left(x, S_{g}(0)\right)}{2 E}\right) & \text { if } & \left|d\left(x, S_{g}(0)\right)\right| \leq E \\
+\frac{2 E}{\pi} & \text { if } & d\left(x, S_{g}(0)\right) \geq E \\
-\frac{2 E}{\pi} & \text { if } & d\left(x, S_{g}(0)\right) \leq-E
\end{array}\right.
$$

where $d\left(x, S_{g}(0)\right)$ is the signed distance from the point $x$ to the initial free surface $S_{g}(0)$. The numerical parameter $E$, called the interface width, is chosen equal to $1.5 \times$ mesh size in the presented simulations. Note that, since $\sin x \approx x$ when $|x| \ll 1, \phi_{0}(x) \approx d\left(x, S_{g}(0)\right)$ in the vicinity of the interface $S_{g}(0)$. Hence, the level-set function $\phi$ corresponds to the signed distance function to the interface in the neighbourhood of this interface. Furthermore, regarding [4], $\phi$ is extended by a constant value (positive or negative) equal to $\pm 2 E / \pi$ outside the narrow band $[-E,+E]$ around the interface $S_{g}$, and is differentiable through the boundaries of this band, i.e., at each $x$ such that $d\left(x, S_{g}\right)= \pm E$.

At any time $t>0, \phi$ is solution, in a neighbourhood of the interface, of the "classical" advection equation,

$$
\frac{\partial \phi}{\partial t}+\mathbf{v}_{\mathbf{s}} \cdot \nabla \phi=0
$$


where $\mathbf{v}_{\mathbf{s}}$ is given by Equation [3], while outside this neighbourhood, a renormalization procedure is applied by enforcing

$$
\|\nabla \phi\|=\sqrt{1-\left(\frac{\pi \phi}{2 E}\right)^{2}}
$$

This approach is similar to the classical renormalization technique consisting in enforcing $\|\nabla \phi\|=1$, but applied to a function $\phi$ of the form [4] (indeed, $\frac{d}{d x} \sin (x)=$ $\left.\cos (x)=\sqrt{1-\sin ^{2}(x)}\right)$. Hence, the advection and the renormalization steps are performed by solving one single equation as described in (Coupez, 2006).

\subsection{Curvature and surface velocity level-set formulation}

By definition, the value of $\phi(x, t)$ gives the position of a point $x$ at a time $t$ with respect to the grain free boundary. Furthermore, and it is a key point for the surface diffusion simulation, both the unit vector $\mathbf{n}_{\phi}$ normal to the interface and the mean curvature $K_{\phi}$ of this interface can be expressed in a level-set way by the two following relations (Sethian, 1999; Osher et al., 2001):

$$
\mathbf{n}_{\phi}=\frac{\nabla \phi}{\|\nabla \phi\|}
$$

and,

$$
K_{\phi}=\nabla \cdot \frac{\nabla \phi}{\|\nabla \phi\|}
$$

where $\|\cdot\|$ denotes the Euclidian norm in $\mathbb{R}^{3}$. Hence, if the level-set function is known, then the curvature can be calculated, at least theoretically. Note that expressions [6] and [7] define the normal vector and the mean curvature in all the computational domain $\Omega$, and not only over the free surface $\{\phi=0\}$. Of course, $\mathbf{n}_{\phi}$ and $K_{\phi}$ vanish outside the narrow band $[-E, E]$ around the interface, while they correspond to the usual normal vector $\mathbf{n}$ and curvature $K$ in the vicinity of the interface (Bruchon et al., 2009a). Following (Burger et al., 2007), the surface diffusion velocity [3] can be rewritten in a level-set form as

$$
\mathbf{v}_{\mathbf{s}}=v_{n} \frac{\nabla \phi}{\|\nabla \phi\|}
$$

and,

$$
v_{n}=C_{0} \frac{1}{\|\nabla \phi\|} \nabla \cdot\left(\|\nabla \phi\| P_{\phi} \nabla K_{\phi}\right)
$$

where the projection matrix [2] is now defined by

$$
P_{\phi}=I-\frac{\nabla \phi}{\|\nabla \phi\|} \otimes \frac{\nabla \phi}{\|\nabla \phi\|}
$$


The velocity [8] is defined in the whole computational domain, and corresponds, in the vicinity of the zero level set of $\phi$, to the surface diffusion velocity. The differential operators (gradient and divergence) involved in Equation [9] are now expressed explicitly with the cartesian coordinates $(x, y, z)$, and can therefore be computed within the context of an Eulerian description of the grains.

\subsection{Curvature - normal velocity stabilized mixed system}

Formulation [9] is discretized by using a finite element approach. The computational domain $\Omega$ is discretized by a simplex mesh $\mathcal{T}_{h}(\Omega)$, that is, a set of tetrahedrons. The unknowns $\phi, K$ and $v_{n}$ are approximated by $\phi_{h}, K_{h}$ and $v_{n h}$, respectively, chosen as being continuous and piecewise linear over $\Omega$. Furthermore, the partition $0=t_{0}<t_{1}<\cdots<t_{\Theta}=\Theta$ of the time interval $[0, \Theta]$ is introduced: $\phi_{h}^{t}, K_{h}^{t}$ and $v_{n}{ }_{h}^{t}$ denote the finite element approximations evaluated at the time $t$.

Regarding Section 3.2, all seems easy: if $\phi_{h}^{t}$ is assumed to be known, then $K_{h}^{t}$ and $v_{n h}^{t}$ could be directly computed by introducing Equations [6], [7] and [9] into the finite element formulation. However, three difficulties appear and must be understood. First, the velocity $v_{n h}$ depends on the fourth-order spatial derivative of $\phi_{h}$. Since $\phi_{h}$ is piecewise linear, the gradient $\nabla \phi_{h}$ is piecewise constant, and the $\mathrm{n}$ th-order derivative $\nabla^{(n)} \phi_{h}$ is identically equal to zero when $n \geq 2$. Equations [7] and [9] must therefore be considered in a weak sense (see Equation [12] and (Burger et al., 2007; Bänsch et al., 2005)). The second difficulty is the nonlinear coupling between the level-set function $\phi$, the curvature and the surface diffusion velocity. We have chosen to treat the velocity in an explicit way in the transport equation [5]. In other words, $\phi_{h}^{t+\Delta t}$ is computed by solving Equation [5] with $\mathbf{v}_{\mathbf{s}}^{t}$, the velocity evaluated on the configuration described by $\phi_{h}^{t}$. Finally, the last difficulty is that the fully explicit scheme which consists in computing successively $K_{h}^{t}$ and $v_{n}{ }_{h}^{t}$, assuming that $\phi_{h}^{t}$ is known, and then to transport $\phi_{h}$ from time $t$ to time $t+\Delta t$, leads to numerical oscillations of the level-set function (Bruchon et al., 2009b).

To overcome these difficulties, the numerical method proposed in this section consists in building a system, the unknowns of which are the curvature $K_{h}^{t}$ and the normal velocity $v_{n}^{t}$ (which is equal to $C_{0} \Delta_{\mathrm{s}} K_{h}$ regarding Equation [3]). In order to introduce implicitly a regularization term in this formulation, the following first-order Taylor's expansion is considered:

$$
\phi_{h}^{t+\frac{1}{2}} \underset{\text { def. }}{=} \phi_{h}^{t}+\frac{\partial \phi_{h}^{t}}{\partial t} \Delta t=\phi_{h}^{t+\Delta t}+o(\Delta t)
$$

Hence, $\phi_{h}^{t+\frac{1}{2}}$ is a first-order approximation of $\phi_{h}^{t+\Delta t}$. Since the level-set function $\phi$ is solution of the transport Equation [5], $\partial \phi / \partial t=-\mathbf{v}_{\mathbf{s}} \cdot \nabla \phi$, and the previous relation can be turned into 


$$
\phi_{h}^{t+\frac{1}{2}}=\phi_{h}^{t}-\mathbf{v}_{\mathbf{s} h}^{t} \cdot \nabla \phi_{h}^{t} \Delta t
$$

Finally, taking into account expression [8] leads to

$$
\phi_{h}^{t+\frac{1}{2}}=\phi_{h}^{t}-v_{n h}^{t}\left\|\nabla \phi_{h}^{t}\right\| \Delta t
$$

The system with the unknowns $K / \Delta_{\mathrm{s}} K$ can now be constructed by considering $\phi_{h}^{t+\frac{1}{2}}$ instead of $\phi_{h}^{t}$ in relation [7] giving the curvature of the discretized level-set function. Hence, at a time $t$, assuming that $\phi_{h}^{t}$ is known, the system in curvature $K_{h}^{t} /$ normal velocity $v_{n h}^{t}$ is given by

$$
\begin{array}{ll}
K_{h}^{t}+\nabla \cdot\left(\frac{\Delta t}{A} \nabla v_{n h}^{t}\right) & =\nabla \cdot\left(\frac{1}{A} \nabla \phi_{h}^{t}\right) \\
v_{n h}^{t}\left\|\nabla \phi_{h}^{t}\right\|-C_{0} \nabla \cdot\left(\left\|\nabla \phi_{h}^{t}\right\| P_{\phi_{h}^{t}} \nabla K_{h}^{t}\right) & =0
\end{array}
$$

The key point of this approach is that using $\phi_{h}^{t+\frac{1}{2}}$ instead of $\phi_{h}^{t}$ to compute the curvature, induces "naturally" an additional term in the left-hand side of the first equation of [11]. This term, coupling $K$ and $v_{n}$, is a regularization term of the form $\Delta v_{n h}$, with the regularization parameter $\Delta t / A$. The parameter denoted by $A$ should be equal to $\left\|\nabla \phi_{h}^{t+\frac{1}{2}}\right\|$. However, to avoid to deal with this nonlinear term, $A$ is simply taken equal to $\left\|\nabla \phi_{h}^{t}-\Delta t \nabla v_{n}^{t-\Delta t}\right\|$. Furthermore, the term $\left\|\nabla \phi^{t}\right\|$ which appears in Equation [10] has been taken identically equal to 1 in this curvature expression.

Since the effective computation of $K_{h}$ and $v_{n h}$ is carried out by using a finite element discretization of [11], the mixed weak formulation of [11] has to be written:

At time $t$, assuming $\phi_{h}^{t}$ known, find $\left(K_{h}^{t}, v_{n}^{t}\right) \in \mathcal{V}_{h} \times \mathcal{V}_{h}$ solution of

$$
\begin{aligned}
& \int_{\Omega} K_{h} \psi_{h} d \Omega-\Delta t \int_{\Omega} \frac{1}{A} \nabla v_{n h}^{t} \cdot \nabla \psi_{h} d \Omega=-\int_{\Omega} \frac{1}{A} \nabla \phi_{h}^{t} \cdot \nabla \psi_{h} d \Omega \\
& \int_{\Omega}\left\|\nabla \phi_{h}^{t}\right\| v_{n h}^{t} \psi_{h} d \Omega+\int_{\Omega} C_{0}\left\|\nabla \phi_{h}^{t}\right\|\left(P_{\phi_{h}^{t}} \nabla K_{h}^{t}\right) \cdot \nabla \psi_{h} d \Omega=0
\end{aligned}
$$

for all $\psi_{h} \in \mathcal{V}_{h}$

Note that one single type of weighting functions $\psi_{h}$ has been used for the curvature equation and for the velocity one, since both curvature and velocity belong to the same functional space $\mathcal{V}_{h}$, the space of the continuous functions which are piecewise linear. Furthermore, previous Equation [12] does not require the enforcement of Dirichlet conditions: since $\phi_{h}$ is constant far from the free boundary, $K_{h}$ and $v_{n h}$ vanish over the mesh boundary. 
To summarize the previous developments, after an initialization step consisting in calculating the initial level-set function by Equation [4], our time-stepping strategy consists, at time $t$ and assuming $\phi_{h}^{t}$ is known, in the computation of the surface velocity by [12] and [8], and in the subsequent transport of the level-set function by solving Equation [5]. A mesh adaptation strategy is also used to obtain an accurate description of the grain free boundary (Bruchon et al., 2009a).

\section{Numerical simulations}

This section presents several direct simulations of change in free surface by surface diffusion, using the numerical strategy developed previously. The presented developments have been implemented in the CIMLIB finite element library. This C++ library, which is highly parallel, is developed at Centre de Mise en Forme des Matériaux (Mines ParisTech, UMR CNRS 7635) by the team of T. Coupez (Digonnet et al., 2003).

\subsection{Change in two grains of the same size by surface diffusion}

Figure 2 shows the temporal change in the zero isosurface of the level-set function corresponding to two spherical grains of radius $R=0.2$. These grains are initially in contact (see Figure 2(a)), though the discretization with a mesh does not allow the grains to be perfectly tangent). Despite the initial "roughness" of the contact area between the grains, and due to the matter diffusion, this surface becomes quickly smooth. The grain volume is well-preserved, and as expected, no shrinkage phenomenon occurs. The radius $x$ of the circular contact area is called the neck radius. Theoretical models, based on geometrical assumptions and established within the context of the sintering process modelling (Rahaman, 2003), predict that the adimensional neck radius $x / R$ behaves as the power $1 / 7$ of the time:

$$
\frac{x(t)}{R}=\left(\frac{56 C_{0}}{R^{4}} t\right)^{1 / 7}=1.78 t^{1 / 7}
$$

where $t^{\prime}$ is an adimensional time, defined by $t^{\prime}=\frac{C_{0}}{R^{4}} t$. Figure 3 shows, in logarithmic scale, the temporal change in the adimensional radius $x / R$, obtained by direct simulation for different values of the grain radius, ranging from 0.1 to 2.5 . Accordingly to the analytical model, the simulation shows that the change in $x / R$ versus $t^{\prime}$, does not depend on the grain radius $R$, and behaves as $t^{1 / 7}$ (curve mentioned as "Simulation, $1 / 7$ " in Figure 3). The only difference between the analytical model and the simulation results, is the coefficient 1.78 provided by the analytical model (curve referred to as "Analytical model, 1/7"). Our simulations provide a coefficient approximatively equal to 1.3 , whatever the time step (ranging from $10^{-6}$ to $10^{-2}$ ) and the mesh size (ranging from $10^{-4}$ to $10^{-2}$, with isotropic or anisotropic remeshing). In Equation (13), the two key parameters which characterize the surface diffusion from the grain surface 
towards the neck, are, on one hand the power $1 / 7$, and on the other hand, the power 4 applied to $R$ in the denominator (Rahaman, 2003). The differences between the simulations and the analytical model may be explained by the geometrical approximations made on the curvature in the analytical model.

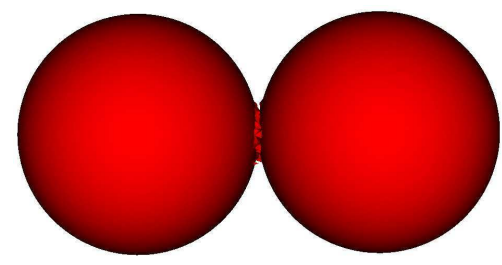

(a) $\mathrm{t}=0$

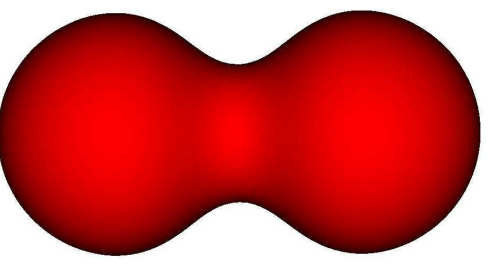

(b) $t=3$

Figure 2. Change in the free boundary $\left\{\phi_{h}=0\right\}$ due to the surface diffusion between two grains of equal size (radius $R=0.1$ )

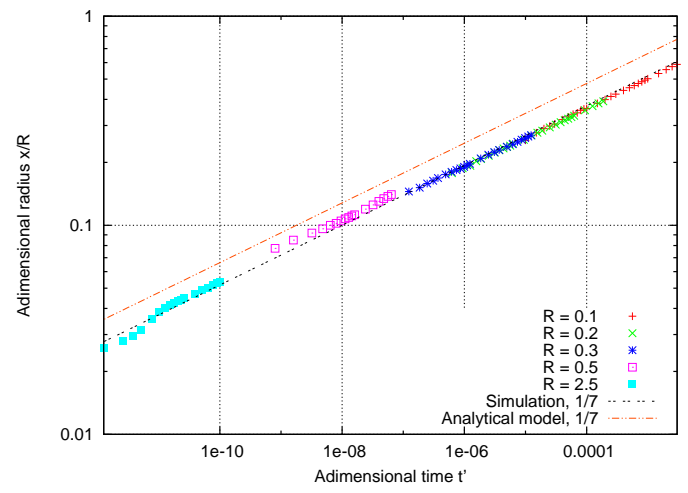

Figure 3. Change in the adimensional neck radius $x / R$ over adimensional time $t^{\prime}$ (logarithmic scale) for different values of $R$, and with $C_{0}=10^{-7}$

\subsection{Change in a small cluster of grains by surface diffusion}

The sintering by surface diffusion of 20 grains (see Figure 4) is investigated in this section. The grain radii vary randomly uniformly between 0.08 and 0.2 , while the grain position is initially randomly uniformly distributed, with the only restrictions that there is no isolated grain, and that two grains in contact must be tangent, as shown in Figure 4(a). The computational domain is the unit-cube, discretized with an unstructured mesh made up of 1,320,000 tetrahedrons (227,000 nodes). This mesh is adapted dynamically, in order to be refined in the vicinity of the free boundary, according to the strategy developed in (Bruchon et al., 2009a) (the mesh size ranges from 0.01 to 0.08 ). As previously, the contact area between two grains are not initially 
well-defined. However, it can be seen when comparing Figures 4(a) and 4(b) that the diffusion smoothes quickly these surfaces. Neck formation is then observed, and the cluster evolves towards an equilibrium state. As expected, no shrinkage phenomenon occurs (the dimensions of the bounding box containing the grains remain constant along the simulation), and the grain volume is well preserved during the simulation as shown in Figure 5. The CPU time of this computationï $i^{1 / 2 i n v o l v i n g} 160$ time steps, is of 165 minutes by using a parallel computing strategy on four cores (Intel Xeon 2.2 $\mathrm{GHz}$ processors).

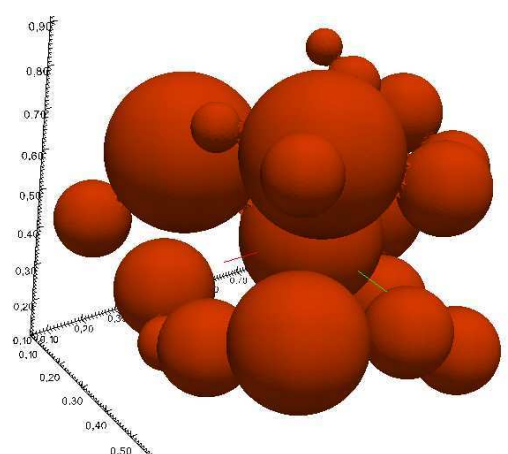

(a) $\mathrm{t}=0$

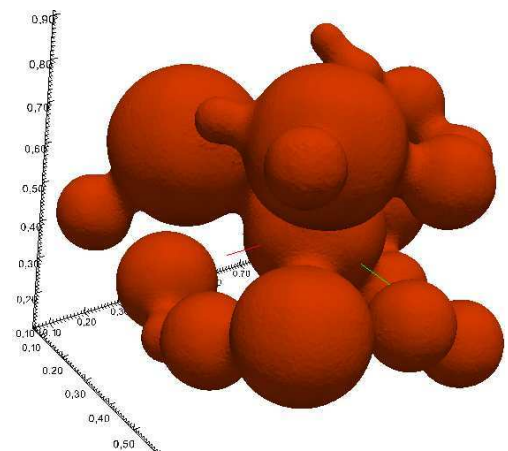

(c) $\mathrm{t}=1.2$

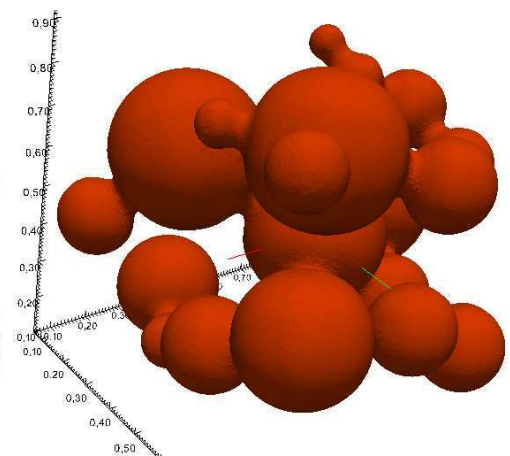

(b) $\mathrm{t}=0.4$

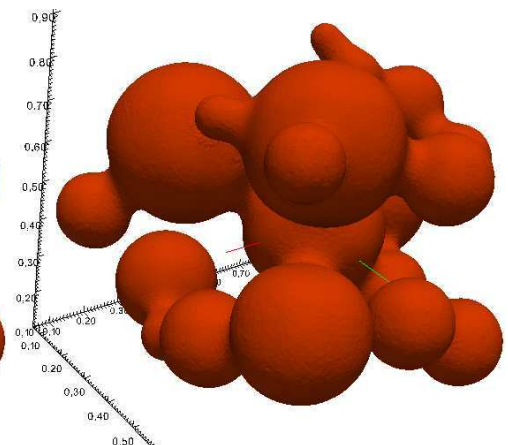

(d) $\mathrm{t}=1.6$

Figure 4. Surface diffusion simulation: change in the free boundary $\left\{\phi_{h}=0\right\}$ of 20 grains of radii randomly uniformly distributed between 0.08 and $0.2\left(C_{0}=10^{-7}\right.$, $\Delta t=10^{-2}$ ) 


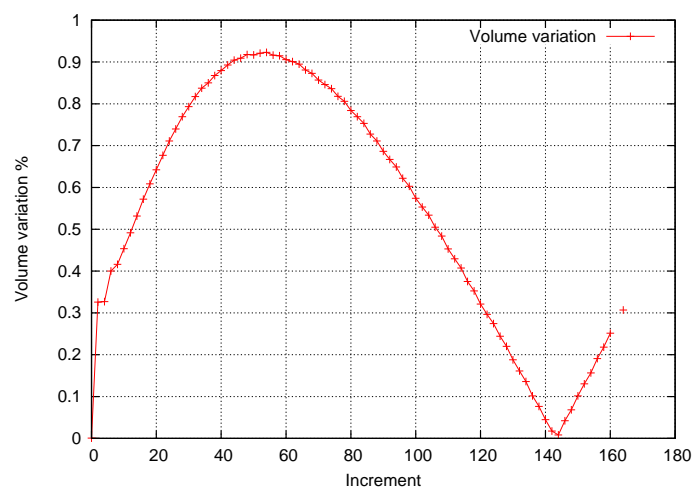

Figure 5. Variation of the grain volume during the simulation (\%)

\section{Conclusion}

Within the general context of the sintering process simulation, a level-set formulation of the surface diffusion problem has been proposed. The main difficulty induced by this approach, the dependence of the surface velocity on the fourth-order spatial derivative of the level-set function, has been overcome by considering a mixed system in curvature / surface Laplacian of the curvature. This formulation induces implicitly a stabilisation term $\Delta v_{n}$ with an associated stabilisation parameter depending on the time step. The resulting discretized formulation is shown to be stable in all our simulations. The accuracy of this approach has been proved by investigating the surface diffusion between two grains and by comparing, in this case, the simulation with an analytical model. Furthermore, the ability of our numerical strategy, to describe the changes occuring in complex geometries, has been shown by detailing a simulation involving 20 grains. The next step in the development of this work is to describe the join separating two particles, in order to model the matter transport by grain boundary diffusion.

\section{References}

Ashby M., “A first report on sintering diagrams”, Acta Metall. Mater., vol. 22, n 3, p. 275-289, 1974.

Bänsch E., Morin P., Nochetto R. H., “ A finite element method for surface diffusion: the parametric case”, J. Comput. Phys., vol. 203, n 1, p. 321-343, February, 2005.

Bernacki M., Chastel Y., Coupez T., Logé R., “Level set framework for the numerical modelling of primary recrystallization in polycrystalline materials", Scripta Mater., vol. 58, n 12 , p. 1129-1132, 2008.

Bouvard D., McMeeking R., " Deformation of interparticle necks by Diffusion-Controlled Creep”, J. Am. Ceram. Soc., vol. 79, n 3, p. 265-672, 1996. 
Bruchon J., Digonnet H., Coupez T., “ Using a signed distance function for the simulation of metal forming processes: Formulation of the contact condition and mesh adaptation. From a Lagrangian approach to an Eulerian approach", Int. J. Numer. Meth. Eng., vol. 78, n ${ }^{\circ}$, p. 980-1008, 2009a.

Bruchon J., Pacquaut G., Drapier S., Valdivieso F., “ Modélisation et simulation du transport de matière par diffusion surfacique à l'aide d'une approche Level-Set", Actes du 9ième Colloque National en Calcul des Structures, Giens (Var, France), p. 517-522, May 25-29, 2009b.

Burger M., Hausser F., Stocker C., Voigt A., “ A level-set approach to anisotropic flows with curvature regularization”, J. Comput. Phys., vol. 225, n 1, p. 183-205, 2007.

Coupez T., "Réinitialisation convective et locale des fonctions Level Set pour le mouvement de surfaces et d'interfaces.", Actes des Journées Activités Universitaires de Mécanique, ISBN 2-9526-8123-8, La Rochelle, p. 33-41, August 31st - September 1st, 2006.

Digonnet H., Coupez T., “ Object-oriented programming for fast-and-easy development of parallel applications in forming processes simulation", Proceedings of the Second MIT Conference on Computational Fluid and Solid Mechanics, 2003.

Osher S., Fedkiw F., “Level Set Methods: An Overview and Some Recent Results”, J. Comput. Phys., vol. 169, n 2, p. 463-502, 2001.

Rahaman M. N., Ceramic processing and sintering, CRC Press, 2003.

Sethian J., Level Sets Methods and Fast Marching Methods, 3 edn, Cambridge Monograph on Applied and Computational Mathematics, 1999. 